and therapeutic management. There is intermediate prevalence of HBV and low prevalence of HIV and HCV in Iran. However, there is little information regarding the number of co-infections of viral hepatitis. The aim of present is study is to establish prevalence of HBV, HDV and HCV co-infection among HIV positive Iranian patients.

Materials and Methods The design of study was cross-sectional during 2010 to 2012. Patients having HIV with co-infection of HBV, HDV or HCV visiting Tehran West Health Centre were enrolled. Serological HBV (HBS Ag, HBC Ab), HDV Ab and HCV Ab were determined in a sub-group of $200 \mathrm{HIV}$ positive patients. HCV RNA PCR(Viral load and genotyping) was determined for all HCV Ab positive patients. Also, HDV Ab was determined for all HBS or HBC Ab positive patients.

Results A total of 200 patients (151 male and 49 female) with a mean age of 33 (2 to 66 years) were evaluated. The prevalence of HBS Ag and HBC Ab was 12\% (21/177) and 24\% (43/177), respectively. HCV Ab was detected in $71 \%(123 / 173)$ out of which $90 \%$ (110/123) were PCR positive with 1b Genotype being the most prominent case. For $43 \mathrm{HBS} \mathrm{Ag}+$ patients, HDV Ab was carried out, 5 of whom were positive and 3 were triple HCV, HBV and HDV coinfection.

Conclusions A high rate of $\mathrm{HIV} / \mathrm{HBV}, \mathrm{HCV}$ co-infection observed in the present study, indicates the need for routine screening of HIV infected patients for viral hepatitis B and C. Such screening could lead into the required treatment for these patients.

\section{P3.217 INTERNATIONAL COMPARISON OF RECENT TRENDS IN THE RATES OF HIV DIAGNOSES AMONG MEN WHO HAVE SEX WITH MEN (MSM)}

doi:10.1136/sextrans-2013-051184.0674

T Foster, N Dickson, P Saxton. University of Otago, Dunedin, New Zealand

Background After a rise in the early 2000s in the number of new HIV diagnoses among MSM in New Zealand, also witnessed in many developed countries, in 2011 the number dropped by $34 \%$ compared to 2010 . To assess relative progress on control we compare trends in HIV diagnosis rates among MSM in developed countries with similarly mature epidemics.

Methods We obtained data on annual HIV diagnoses among MSM between 2003-2011 from 17 developed countries (Australia, Belgium, Canada, Denmark, Finland, France, Iceland, Ireland, Netherlands, New Zealand, Norway, Spain, Sweden, Switzerland, Germany, UK, US). We reallocated those with unknown means of infection according to the countries' pattern of known causes, and used countries' adjustment for delayed reporting where available. The diagnosis rate was derived using the population of men aged 15-64.

Results

- New Zealand has low rates compared to most countries of Western Europe, North America and Australia, and are comparable with those of Scandinavia

- All counties except New Zealand, Iceland and Canada, experienced a slight overall rise in diagnosis rates in the period 2003-2011

- Over the past four years there has been a:

- Slight trend upwards in UK, Belgium, France, Australia, Ireland

- No clear trend in Spain, Canada, Germany, Denmark, Norway

- Slight trend downwards in New Zealand, the Netherlands, Sweden, Finland, and possibly Iceland

- Clear trend downwards in Switzerland.

Conclusions New Zealand has a low rate of HIV diagnoses, relative to many other developed countries. Our drop in 2011 HIV is encouraging but not unique. Limitations of this study are that the data are of diagnosis not infection rates, are influenced by patterns of testing, immigration and emigration, and dual modes of transmission, and the proportion who are MSM may vary between countries. Factors relating to recent trends should be explored.

\section{P3.218 INCREASING TREND OF HIV/AIDS AMONG ARAB AND JEWISH MALES IN ISRAEL, 1986-2010}

doi:10.1136/sextrans-2013-051184.0675

'Z Mor, ${ }^{2} \mathrm{E}$ Grayeb, ${ }^{3} \mathrm{~A}$ Beany, ${ }^{4} \mathrm{G}$ Grotto. 'Ministry of Health, Ramla, Israel; ${ }^{2}$ Hadassah Medical Center, Jerusalem, Israel; ${ }^{3} B n a i$ Zion Medical Center, Haifa, Israel; ${ }^{4}$ Ministry of Health, Jerusalem, Israel

Background HIV/AIDS-burden in Israel is low ( $~ 6$ cases:100,000 population), while sub-populations characterised by high-risk behaviours are affected disproportionally. This study aims to compare HIV/AIDS-burden in males between Israeli Jews and Arabs, which are the biggest monitory in Israel.

Methods The National HIV/AIDS Registry (NHAR) was the source for HIV/AIDS-infection records, while the Israeli Central Bureau of Statistics was used to determine groups-specific disease rates.

Results Between 1986 and 2010, 3,499 HIV/AIDS-infected males were reported: 3,369 (96.3\%) Jews and 130 (3.7\%) Arabs, in an average annual incidence of 5.5 and 0.8 per 100,000 populations, respectively, $p=0.05$. Of all Jews, 1,018 (29.9\%) were born in Ethiopia, while 2,389 were Jews who were not Ethiopian-born (JNE). Most Arabs ( $\mathrm{N}=99$, 74.8\%) were Muslims, followed by 21 (16.2\%) Christians and 13 (10\%) Druze. AIDS rather than HIV upon reporting was diagnosed in $568(23.8 \%)$ of JNE and $31(23.8 \%)$ of the Arabs, $p=1$. The most affected age-group among JNE was 25-34 and in Arabs 20-24, and the respective cumulative death rates were $24.9 \%(\mathrm{~N}=594)$ and $32.5 \%$ $(\mathrm{N}=40), \mathrm{p}=0.1$. The point-prevalence in 2010 was 58.4 and 11.4 per 100,000 for JNE and Arabs, and in adults aged 15-49, was 98.0 and 20.4 per 100,000, respectively. In Muslims, Christians and Druze, the point-prevalence was 4.2,11.2 and 7.1 per 100,000; and in adults aged 15-49 was 20.4, 52.6 and 21.6, respectively.

The most common risk-groups among JNE was MSM (N =1,223, $51.2 \%$ ), followed by IVDU ( $\mathrm{N}=661,27.7 \%)$; while among Arabs was MSM ( $\mathrm{N}=63,48.1 \%)$, followed by heterosexuals $(\mathrm{N}=36$, $27.3 \%)$.

Conclusions HIV/AIDS-burden in Arab males was significantly lower than in Jews. Among Arab-males, HIV/AIDS-burden was highest in Christians than in Druze and Muslims. The proportion of MSM of all males, regardless of their religion is increasing. Interventions aiming to prevent further HIV-transmission should address cultural, linguistic and behavioural characteristics.

\section{P3.219 A 20-YEARS RETROSPECTIVE COHORT STUDY OF HIV SITUATION AMONG HILL TRIBE VULNERABLE POPULATION THAILAND}

doi:10.1136/sextrans-2013-051184.0676

A Tawatchai. School of Health Science, Mae Fah Luang University, Chiang Rai, Thailand

Thailand had been reported as the highest HIV/AIDS epidemic area. Most of HIV/AIDS had been reported from the north of Thailand. Northern Thailand is the favourite living places of hill tribe people who migrated from the south of China through Myanmar last 150 years ago. 600,000 people were living in these areas with differences of culture and life styles. The objective aimed to investigate the situation of HIV/AIDS among hill tribe marginalised and vulnerable population.

Methods The retrospective cohort study was conducted. The systematic data extraction from the medical records in 16 hospitals in northern Thailand during 1990-2010 was performed. The six main hill tribe people: Akha, Lau, Karen, Yao, Kmong, and Lisu were the target population. Chi square test was analysed. 
Results Totally 3,130 cases were recruited into the study. $54.6 \%$ were males, the first case had been reported in 1990, and the highest incident case had been reported in the year 2004 with 461 cases followed by 2005 (343), and 2006(302) respectively. The highest cumulative case had been reported from Mae Fah Luang Hospital (25.8\%), followed by Mae Suai hospital (18.8\%). 46.0\%were Akha, 19.7\%were Lahu, and $9.5 \%$ were Yao. 38.8\%were $31-40$ years old, followed by 21-30 years old(33.6\%), and $41-50$ years old (13.4\%). $44.4 \%$ were agriculture, $32.0 \%$ were employee. $91.6 \%$ were infected by sexual intercourse, $5.7 \%$ were mother to Child. $24.0 \%$ were receiving ARV, $30.7 \%$ were receiving OI treatment, and $9.5 \%$ were tested CD4 level. Male had higher of survival rate than female ( $p$-value $>0.001$ ), and male were younger than female at the age of infection ( $p$-value $>0.001$ ). There was statistically significant difference of mode of infection by tribe ( $p$-value $>0.001$ ).

Conclusion Specific health education programmes and empower them for using condom are needed to setting up for HIV/AIDS prevention and control among hill tribe people in Thailand.

\section{P3.220 INCIDENCE AND PREVALENCE OF HIV INFECTIONS AMONG FISHERMEN AROUND LAKE VICTORIA IN KISUMU KENYA}

doi:10.1136/sextrans-2013-051184.0677

${ }^{1,2,3} \mathbf{R} 0$ Ondondo, ${ }^{3} \mathrm{Z}$ W Ng'ang'a, 'S Mpoke, ' M K Kiptoo, 1,2,3 E A Bukusi. ' ${ }^{\prime}$ Kenya Medical Research Institute, Nairobi, Kenya; ${ }^{2}$ n Collaboration with University of California San Francisco, San Francisco, CA, United States; ${ }^{3}$ Jomo Kenyatta University of Agriculture and Technology, Nairobi, Kenya

Background Men with high-risk sexually behaviour are important drivers of HIV/STI infections in the general population. Prevention of HIV/STIs among such men could potentially reduce these infections especially among women - who bear the greatest HIV/STIs disease burden in sub Saharan Africa. We sought to understand immunovirology of HPV infections among fishermen. This evaluation highlights the rate of HIV infections in this population.

Methods Three hundred fishermen were recruited and followed up every 3 months for 1 year. HIV, syphilis serology, CD4/CD8 and complete blood count were evaluated and a demographic questionnaire administered. Data was analysed by SPSS ver18.

Results The 300 men recruited into this study had mean age of 28 years, $76 \%$ were married and $57 \%$ had only basic education. The mean for age of sexual debut and lifetime sexual partners was 15 years and 10 respectively. They had a modal sexual activity of 3 times/week and a mean of 3 rounds/sexual act. Sixty one (20\%) had practised oral sex. Thirty six (12\%) and $80(27 \%)$ of men washed their genitals, before and after sex respectively. A significant number of men; 183 (61\%) P < 0.01 and 118 (39\%) P < 0.05, never used condoms with their regular sexual partners and new sexual partners respectively. They had a mean CD4 and white blood cell (WBC) count of 830 cells $/ \mathrm{ml}$ and $5.6 \times 10^{9} / \mathrm{L}$ of blood. Baseline HIV and syphilis prevalence was $23 \%$ and $9 \%$ respectively. The HIV incidence during the 1 year follow-up was $4.2 \%$. Fishermen HIV burden was over 3 times the Kenyan national HIV prevalence of $7 \%$.

Conclusion Fishermen in this context, comprise a young sexually high-risk, highly-migratory population with high HIV incidence and prevalence. Low condom use coupled with concurrent multiple sexual partnerships make them a potential key population who require targeted prevention strategies to reduce HIV/STI infections and transmission.

\section{P3.221 ORAL AND INJECTABLE HORMONAL CONTRACEPTION DECREASE RISK OF BACTERIAL VAGINOSIS BUT ORAL CONTRACEPTION MAY INCREASE RISK OF VAGINAL CANDIDIASIS: A SYSTEMATIC REVIEW OF PUBLISHED AND UNPUBLISHED DATA}

doi:10.1136/sextrans-2013-051184.0678
J H van de Wijgert, ${ }^{2} \mathrm{M}$ C Verwijs, ${ }^{3} \mathrm{~A}$ Norris Turner, ${ }^{4} \mathrm{C}$ S Morrison. ${ }^{1}$ University of Liverpool, Institute of Infection and Global Health, Liverpool, UK; ${ }^{2}$ Amsterdam Institute of Global Health and Development (AIGHD), Amsterdam, The Netherlands; ${ }^{3} T$ The Ohio State University, Division of Infectious Diseases, Columbus, OH, United States: ${ }^{4} \mathrm{FH} 360$ Clinical Sciences, Durham, NC, United States

Background A recent World Health Organization (WHO) technical consultation concluded that combined oral contraception (COC) does not increase HIV acquisition in women, but the evidence for depot medroxyprogesterone acetate (DMPA) is conflicting. Significant evidence suggests that bacterial vaginosis (BV) and vaginal candidiasis, both representing an 'unhealthy' vaginal microbiome, increase HIV acquisition in women.

Methods We conducted a systematic review using the PRISMA 2009 guidelines, and re-analysed the Hormonal Contraception and HIV Acquisition (HC-HIV) study, to evaluate the effect of HC use on the vaginal microbiome. Vaginal microbiome outcomes included BV by Nugent scoring, vaginal candidiasis by culture or $\mathrm{KOH}$ wet mount, and microbiome compositions as characterised by molecular techniques.

Results Our review of 36 eligible studies found that COC and DMPA use reduce BV by $10-20 \%$ and $18-30 \%$, respectively. The $\mathrm{HC}-\mathrm{HIV}$ data showed that COC and DMPA use also reduce intermediate microbiota (Nugent score of $4-6$ ) by $11 \%$ for each. In contrast, COC use (but not DMPA use) may increase vaginal candidiasis; 7 of 12 studies reported a statistically significant increase in vaginal candidiasis, 2 reported a positive association approaching significance, 2 reported no association, and one reported a statistically significant reduction. Evidence for a reduction of BV risk in $\mathrm{HC}$ users is much stronger than evidence for a potential increased candidiasis risk in COC users: the quality of the BV studies was higher and the results more consistent. Molecular vaginal microbiome studies $(n=4)$ confirm that high oestrogen levels favour a vaginal microbiome composition dominated by 'healthy' Lactobacillus species; the effects of progesterone on the microbiome are less clear.

Conclusions The hypothesis that DMPA use may increase HIV risk by increasing BV or vaginal candidiasis risk is not supported by the evidence. COC use may predispose for vaginal candidiasis, but is not believed to be associated with increased HIV acquisition

\section{P3.222 HIGH RISK HUMAN PAPILLOMAVIRUS VIRAL LOAD AND PERSISTENCE AMONG HIV-NEGATIVE AND HIV-POSITIVE MEN}

doi:10.1136/sextrans-2013-051184.0679

${ }^{1} \mathbf{M}$ K Grabowski, ${ }^{2,1} \mathrm{R}$ H Gray, ${ }^{2,3} \mathrm{D}$ Serwadda, ${ }^{4} \mathrm{G}$ Kigozi, ${ }^{1,5} \mathrm{P}$ E Gravitt, ${ }^{2} \mathrm{~F}$ Nalugoda, ${ }^{1,2,6} \mathrm{~S}$ J Renyolds, ${ }^{1,2} \mathrm{M}$ Wawer, ${ }^{1,6} \mathrm{~T}$ C Quinn, ${ }^{1,7} \mathrm{~A}$ A R Tobian. ' ${ }^{1}$ Johns Hopkins, Baltimore, MD, United States; ${ }^{2}$ Rakai Health Sciences Program, Entebbe, Uganda; ${ }^{3}$ School of Public Health, Makerere University, Kampala, Uganda; ${ }^{4}$ Rakai Health Sciences Program, En, Uganda; ${ }^{5}$ Perdana University Graduate School of Medicine, Serdang, Malaysia; ${ }^{6}$ Division of Intramural Research, National Institute of Allergy and Infectious Diseases, National Institutes of Health, Bethesda, MD, United States; 'Rakai Health Sciences Program, Entebbe, Austria

Background High-risk human papillomavirus (HR-HPV) viral load is associated with transmission and persistence in women. It is unknown whether viral load is associated with HR-HPV persistence in HIV-negative or HIV-positive men.

Methods 703 HIV-negative and 233 HIV-positive heterosexual men participated in a male circumcision trial in Rakai, Uganda. Penile swabs were tested at enrollment and 6,12 and 24 months for HR-HPV using the Roche HPV Linear Array, which provides a semiquantitative measure of HPV shedding by hybridization band intensity (graded:1-4). Prevalence risk ratios (PRR) were used to estimate the association between HR-HPV viral load and persistent detection of type-specific HR-HPV infection. 